\title{
Body Composition and its relation to Cardio-Respiratory Fitness of Pre-school children.
}

Hany M. Zakaria', Eman M. Araby ${ }^{2}$ \& Ehab M. Emadeldin ${ }^{3}$

1. Assistant Professor and Head of the Department of Educational Sciences and Psychological - Faculty of Physical Education for Boys University of Benha - Egypt, Postal Code 13511. E-mail : Hany.Desouky@ Fped.bu.edu.eg

2. Assistant Professor of Public Health and Community Medicine, Faculty of Medicine, Benha University, Egypt, Postal Code 13511. E-mail :eman.attia@fmed.bu.edu.eg

3. Lecturer of Physical Health Sciences, Faculty of Physical Education for Boys, Benha University, Egypt, Postal Code 13511. E-mail :

Ehab.Emad@Fped.bu.edu.eg Introduction:

Noncommunicable diseases (NCDs) are by far the leading cause of death in the world and their impact is steadily growing. This largely invisible epidemic is more serious in low- and middle-income countries, where $80 \%$ of all NCDs occur. The main causes of NCDs are a small set of modifiable risk factors like unhealthy diet, physical inactivity and tobacco use. Elimination of these modifiable risk factors would prevent $80 \%$ of premature heart disease, $80 \%$ of premature stroke, $80 \%$ of type 2 diabetes and $40 \%$ of cancer. ${ }^{1}$ Children are not immune to this burden. Overweight children, and obesity and type 2 diabetes in children and adolescents are increasing problems. Overweight and obese children are likely to stay obese in adulthood and more likely to develop diabetes and cardiovascular diseases at a younger age. ${ }^{2}$ Many low- and middle-income countries are now facing a "double burden" of infectious diseases and undernutrition. Children in those countries are exposed to high-fat, high-sugar, high-salt, energy-dense, and micronutrient-poor foods, which tend to be lower in cost but also lower in nutrient quality. These dietary patterns, in conjunction with lower levels of physical activity, result in sharp increases in childhood obesity while undernutrition issues remain unsolved. ${ }^{3}$ In Eastern Mediterranean Region (EMR), the prevalence of overweight among children aged 0-5 years was higher in most low- and middle-income countries compared to high income countries. ${ }^{4}$ In Egypt, The proportion of children under-five years, who are overweight in both genders during 2014; was $14.9 \%$ while the proportion of moderately and severely underweight was $5.5 \%$. For children under 5 years of age, overweight is weight-for-height greater than 2 standard deviations above WHO Child Growth Standards median; and obesity is weight-forheight greater than 3 standard deviations above the WHO Child Growth 
Standards median. ${ }^{5}$ Promoting healthy physical activity (PA) behaviors in children between the ages of 0 to 5 years has immediate impacts on the health and well-being of children and serves as a powerful strategy to prevent or minimize the occurrence of chronic diseases in later life Although children are naturally active, their PA levels generally fall below the current WHO recommendations ${ }^{6}$.

Body composition(BC)(fatness) are key components that influence health outcomes, and its measurement is increasingly considered valuable in clinical practice. Anthropometry is considered a simple, inexpensive and easy-to-use method in epidemiological studies. BMI and skinfolds measurements provide excess weight information, but BMI indicates total BF and the sum of skinfolds indicates BF distribution. ${ }^{7}$ Nowadays assessments are more often based on the analyses of body composition and fat distribution. ${ }^{8-13}$ Reliable and valid approach for estimation of human body composition is a technique known as bioelectrical impedance (BIA). This method is safe, noninvasive, provides rapid measurements, requires little operator skill and subject cooperation, and is portable. ${ }^{14}$ Cardio-respiratory fitness denotes the general extent of metabolic processes occurring in the human body.$^{15} \mathrm{CRF}$ is indicated by maximal oxygen uptake per minute $\left(\mathrm{VO}_{2} \max \right)$ as the international standard and primarily determined by the efficiency of mechanisms supplying active muscles with oxygen from the air. ${ }^{16}$ The basic unit of measuring maximal oxygen uptake is its absolute value expressed in liters or milliliters per minutes. However, the absolute value is highly affected by body mass (BM), so it is often expressed as relative value in milliliter $/ \mathrm{kg} /$ minutes.${ }^{17}$ Other factors affecting CRF include BM and BC. ${ }^{18}$ $\mathrm{BC}$ and $\mathrm{VO}_{2}$ max are essential indicators of good physical fitness. ${ }^{19}$ The relationship between Muscle Mass( $\mathrm{MM})$ and $\mathrm{VO}_{2} \max$ is directly proportional and inversely proportional to BF\%. ${ }^{20}$ Improvement in some BC components such as the body mass index (BMI), body $\mathrm{MM}$ and $\mathrm{BF} \%$ as result by the exercise can improve $\mathrm{VO}_{2} \max .{ }^{21}$ In epidemiological studies involving young people, the most common test for assessing cardiorespiratory fitness was the 20-m shuttle run test (Beep Test), or modifications of this test. ${ }^{22}$ The 20$\mathrm{m}$ shuttle run can provide accurate predictions of $\mathrm{VO}_{2} \mathrm{max}$ with very strong correlations with actual $\mathrm{VO}_{2}$ max. ${ }^{23}$ The $\mathrm{VO}_{2}$ max can then be estimated from the score obtained in this test from the published tables in Ramsbottom, Brewer, \& Williams. ${ }^{24}$ The study rational is to identify the physical composition and its relation to the CRF of pre-school children.

\section{Methodology:}

It is a correlational cross-sectional study to investigate the relationship between body composition of pre-school children and their CRF. The study 
comprised (400) children chosen randomly by cluster sampling technique . Qalyubiya governorate includes (12) educational directorates and we selected two (Benha \& Toukh) randomly. Pre-school children in both directorates represent $16.7 \%$ of total pre-school children in the governorate . Each directorate includes number of governmental Kindergartens. Finally , we selected 8 Kindergartens and our sample unit was a class from each one. Three hundred children from six governmental Kindergartens in Benha (Urban), and one hundred from Toukh (semi-urban).

Inclusion criteria:

1. All selected children are a pre-school ones with age range (4 to less than 6 years).

2. All included children are under umbrella of health insurance.

\section{Exclusion criteria:}

1. Children with positive family history of hereditary disease

2. Children with chronic cardiac or respiratory disorders ( congenital heart disease , allergy \& asthma)

3. Handicapped or children with bone fractures

\section{Ethical consideration:}

Administrative approval was obtained from related educational directorates before study conduction and written informed consents were obtained from children guardians before participation in the study. They were informed about the study aim and procedures. We emphasized confidentiality of data and safety of children.

\section{Measurements procedures:}

All variables were measured between ( $8-11 \mathrm{am}$ ) by trained researchers using the following equipment:

A. Calibrated weight \& height scales: Weight was measured using a digital anthropometric scale, graded from 0 to $150 \mathrm{~kg}$. The children were lightly dressed and without shoes. Height was measured using a metric tape fixed on the wall and extended from the bottom upwards, with the children kept in a vertical position, with feet and trunk leaning against the wall.

B. Digital Blood Pressure Monitor : Diastolic and systolic blood pressure levels (DPB and SBP) were determined by an automatic blood pressure monitor. The children must be sitting, at least 5 minutes rest, with the arm supported. Mean arterial pressure (MAP) was calculated using the following formula: DBP $+[0.333 \times($ SBP-DBP $)]$.

C. Bodystat QuadScan 4000 : To monitor body composition parameters like Fat mass, Fat free mass, BMI,....etc. by measuring hand-to-foot whole body electrical impedance. Children were instructed to remove their 
shoe and sock from their right foot, remove any watches or bracelets on the right wrist which may impede the correct placing of electrodes and to lie in the supine position for 5 minutes before taking the measurements. Legs and arms are separated out so they are not in contact with any other part of the body. The area of the skin where the electrodes are to be attached was thoroughly wiped using alcohol wipes ${ }^{25}$

D. Ergospirometry: To assess the efficiency of the circulatory \& respiratory systems, especially the maximum oxygen consumption.

E. Complete Spirostik : To measure vital capacity, Forced Vital

Capacity\& Forced Expiratory Volume in the ${ }^{1 \text { st }}$ second \& Peak Expiratory Flow.

Pilot study:

The researchers carried out body composition measurements and cardiorespiratory measurements on the sample of (60) children in the period from 1/10/2019 to 14/10/2019, with the aim of:

1. Ensure the safety of the used devices.

2. Determine the time required for each measurement.

3 . Determine the optimal system for the sequence of measurements.

4 . Determine the variables needed by the researchers of measurements (physical composition, cardio-respiratory fitness).

\section{The Experience of Basic Research}

The researchers conducts the body composition and cardio-respiratory measurements on the sample of (400) children in the period from in the period from $20 / 1 / 2019$ to $19 / 12 / 2019$.

Data management:

Data were analyzed using SPSS, v.25.0 for Windows (SPSS Inc, Chicago, USA), and the significance level was set at $p<0.05$. The data are shown in descriptive statistics for mean and standard deviation (SD). Tests of normal distribution and homogeneity (Kolmogorov-Smirnov and Levene's, respectively) were conducted on all data before analysis. Differences between sex and age groups were analyzed using analysis of variance (ANOVA) adjusting by Bonferroni test; additionally, differences between weight status were analyzed with ANOVA using as covariables: age and sex. Finally, a Pearson correlation analysis was performed between physical fitness tests with BMI adjusted for age ,Body fat, fat \%. Multilinear regression analysis for detection of significant predictors of CRF will be done.

Results:The study included 400 (200 boys \& 200 girls) pre-school children with mean age $4.7 \pm 0.7$ years, height $115.2 \pm 5.9 \mathrm{~cm}$, weight $23.3 \pm 3.2 \mathrm{~kg}$ and BMI17.5 $\pm 1.7 \mathrm{~kg} / \mathrm{m}^{2}$ and according to WHO classification of BMI for pre-school children with adjusted age \& sex using "CDC: BMI Percentile 
Calculator for Child and Teen" we found that 172 out of 400 (43\%) of the study group were obese ( 94 boys \& 78 girls), 86 (21.5\%) over-weight (31 boys \& 55 girls) while 21 (5.3\%) under-weight ( 9 boys \&12 girls). The remaining children 121 (30.2\%) normal weight (67 boys \& 54 girls). There were significant differences between boys \& girls regarding body composition variables . girls were taller (115.8 $\pm 6.3 \mathrm{vs} .114 .5 \pm 5.4)$ with more body fat content $(5.8 \pm 1.23$ vs. $4.9 \pm 1.25)(\mathrm{p}<0.05)$ \& higher body fatness \% (24.6 \pm 2.6 vs. $21.3 \pm 2.7)(\mathrm{p}<0.01)$. while boys had higher lean weight ( $18.2 \pm 2.1$ vs. $17.7 \pm 2.1)$ \& dry lean weight ( $3.96 \pm 5.4$ vs. $3.94 \pm 5.7$ ) $(p<0.05)$, in addition to higher water content $(14.2 \pm 1.6$ vs.13.7 \pm 1.6$)$ \& lean weight \% (78.7 $\pm 2.8 v s .75 .4 \pm 2.6)(p<0.01)$. Regarding BMI classification ,girls represented (64\%) of over-weight category while boys represented $(54.7 \%)$ of obese category. The higher percentage of under- weight was among girls $(57.1 \%)$. All the previous finding were statistically significant $\left(X^{2}\right.$ test:10.7 \& p<0.05). Table (1) \& Figure (1)

Table (1): Physical body composition measurements

\begin{tabular}{|l|c|c|c|c|c|}
\hline Variables & $\begin{array}{l}\text { Mean } \pm \\
\text { SD }\end{array}$ & Boys (200) & Girls (200) & $\begin{array}{c}\text { T- } \\
\text { test }\end{array}$ & $\begin{array}{c}\text { P } \\
\text { value }\end{array}$ \\
\hline Age(Y) & $4.7 \pm 0.7$ & $4.7 \pm 0.77$ & $4.6 \pm 0.64$ & 0.48 & $>0.05$ \\
\hline Height(cm) & $\begin{array}{c}115.2 \pm \\
5.9\end{array}$ & $114.5 \pm 5.4$ & $115.8 \pm 6.3$ & 1.96 & ${ }^{*}<0.05$ \\
\hline Weight(kg) & $23.3 \pm 3.2$ & $23.1 \pm 3.2$ & $23.5 \pm 3.24$ & 0.99 & $>0.05$ \\
\hline BMl(kg/m2) & $17.5 \pm 1.7$ & $17.6 \pm 1.8$ & $17.5 \pm 1.6$ & 0.62 & $>0.05$ \\
\hline $\begin{array}{l}\text { Body Fat } \\
\text { (kg) }\end{array}$ & $5.4 \pm 1.3$ & $4.9 \pm 1.25$ & $5.8 \pm 1.23$ & 6.6 & ${ }^{*}<0.05$ \\
\hline $\begin{array}{l}\text { (lean } \\
\text { weight)(kg) }\end{array}$ & $17.9 \pm 2.2$ & $18.2 \pm 2.1$ & $17.7 \pm 2.1$ & 2.34 & ${ }^{*}<0.05$ \\
\hline $\begin{array}{l}\text { Water } \\
\text { content (L) }\end{array}$ & $3.9 \pm 0.6$ & $14.2 \pm 1.6$ & $13.7 \pm 1.6$ & 3.0 & ${ }^{* *}<0.01$ \\
\hline dry lean(kg) & $14 \pm 1.6$ & $3.96 \pm 5.4$ & $3.94 \pm 5.7$ & 0.39 & ${ }^{*}<0.05$ \\
\hline $\begin{array}{l}\text { Body } \\
\text { fatness\% }\end{array}$ & $22.97 \pm 3.1$ & $21.3 \pm 2.7$ & $24.6 \pm 2.6$ & 12.2 & ${ }^{* *}<0.01$ \\
\hline $\begin{array}{l}\text { Body lean } \\
\%\end{array}$ & $77.03 \pm 3.1$ & $78.7 \pm 2.8$ & $75.4 \pm 2.6$ & 12.2 & ${ }^{* *}<0.01$ \\
\hline $\begin{array}{l}\text { Waist } \\
\text { circum.(cm) }\end{array}$ & $62.6 \pm 4.4$ & $62.8 \pm 4.6$ & $62.4 \pm 4.2$ & 1.03 & $>0.05$ \\
\hline $\begin{array}{l}\text { Hip } \\
\text { circum.(cm) }\end{array}$ & $67.63 \pm 4.1$ & $67.8 \pm 4.2$ & $67.5 \pm 4.1$ & 0.95 & $>0.05$ \\
\hline
\end{tabular}




\begin{tabular}{|c|c|c|c|c|c|}
\hline $\mathrm{W} / \mathrm{H}$ ratio & $\begin{array}{c}0.92 \pm \\
0.04\end{array}$ & $0.928 \pm 0.046$ & $0.927 \pm 0.048$ & 0.1 & $>0.05$ \\
\hline
\end{tabular}

significant $(p<0.01)$

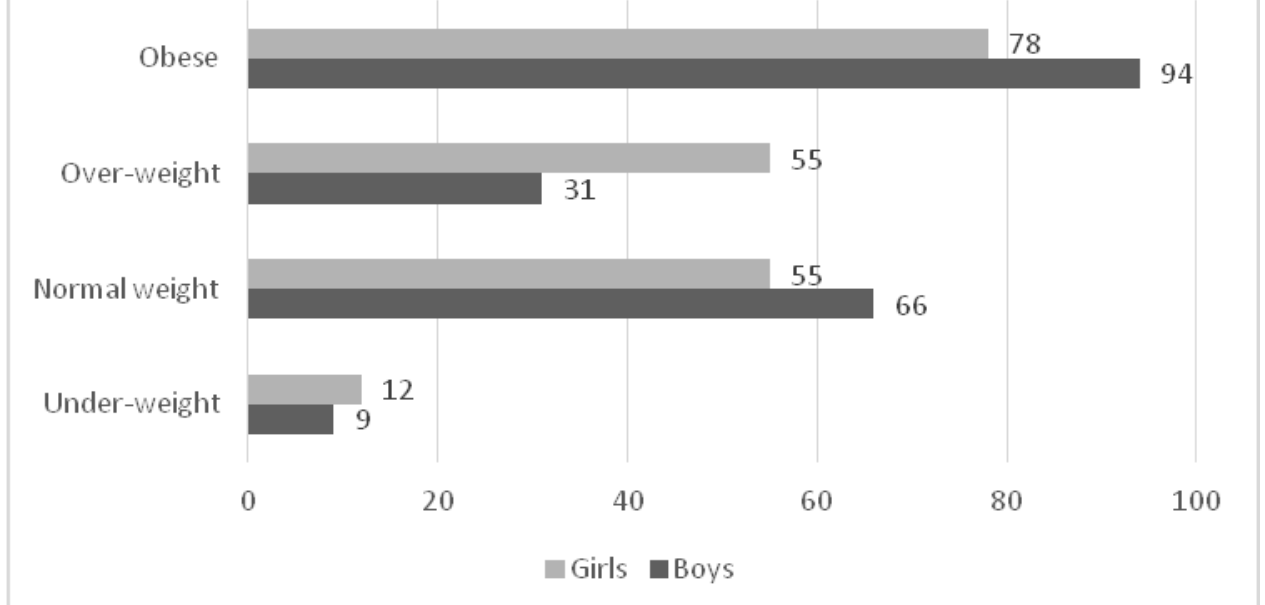

Fig.1 Classification of the studied group according to BMI $\left(X^{2}\right.$ test:9.6 \& $\left.{ }^{*} p<0.05\right)$

Table (2) shows comparison between boys \& girls regarding CRF. $\mathrm{VO}_{2} \mathrm{max}$ which is essential indicator of good physical fitness was lower than the normal range for the same age group, which reflects poor CRF of the studied children especially girls. Moreover, girls showed significantly higher systolic , diastolic and mean arterial pressure than boys even if their findings were within the normal reference ranges for the same age group .

Table (2): Cardio- respiratory measurements of the studied group

\begin{tabular}{|c|c|c|c|c|c|}
\hline $\begin{array}{l}\text { Cardio- } \\
\text { respiratory } \\
\text { variables }\end{array}$ & $\begin{array}{c}\text { Total } \\
(400) \\
\text { Mean } \pm \\
\text { SD }\end{array}$ & $\begin{array}{l}\text { Boys } \\
(200) \\
\text { Mean } \pm \\
\text { SD }\end{array}$ & $\begin{array}{c}\text { Girls } \\
(200) \\
\text { Mean } \pm \\
\text { SD }\end{array}$ & $\begin{array}{c}\text { T- } \\
\text { test }\end{array}$ & $\begin{array}{c}\mathbf{P} \\
\text { value }\end{array}$ \\
\hline$(\mathrm{IVC})(\mathrm{L})$ & $1.2 \pm 0.6$ & $1.66 \pm 0.49$ & $0.72 \pm 0.15$ & 25.6 & ${ }^{* *}<0.01$ \\
\hline$(\mathrm{FVC})_{(L)}$ & $1.2 \pm 0.4$ & $1.47 \pm 0.34$ & $0.83 \pm 0.10$ & 25.2 & ${ }^{* *}<0.01$ \\
\hline $\mathrm{FEV}_{1(\mathrm{~L})}$ & $1 \pm 0.4$ & $1.32 \pm 0.3$ & $0.76 \pm 0.11$ & 24.4 & ${ }^{* *}<0.01$ \\
\hline PEF & $1.3 \pm 0.4$ & $1.56 \pm 0.29$ & $1.02 \pm 0.11$ & 24.1 & ${ }^{* *}<0.01$ \\
\hline $\begin{array}{l}\mathrm{Vo}_{2} \mathrm{max} . \\
(\mathrm{ml} / \mathrm{Ka} / \mathrm{min})\end{array}$ & $20.7 \pm 3.9$ & $23.87 \pm 2.8$ & $17.5 \pm 1.33$ & 28.9 & ${ }^{* *}<0.01$ \\
\hline $\mathrm{SBP}(\mathrm{mm} \mathrm{Hg})$ & $\begin{array}{c}102.4 \pm \\
5.2\end{array}$ & $98.3 \pm 4.2$ & $106.5 \pm 1.4$ & 28.7 & ${ }^{* *}<0.01$ \\
\hline $\mathrm{DBP}(\mathrm{m}$ & $\begin{array}{l}70.8 \\
\pm 4.4\end{array}$ & $67.3 \pm 3.1$ & $74.4 \pm 1.5$ & 25.9 & ${ }^{* *}<0.01$ \\
\hline $\mathrm{MAP}(\mathrm{mm} \mathrm{Hg})$ & $81.4 \pm 4.6$ & $77.6 \pm 3.5$ & $85.1 \pm 1.4$ & 27.1 & ${ }^{* *}<0.01$ \\
\hline
\end{tabular}




\begin{tabular}{l|l|l|l|l|l|}
\hline $\begin{array}{l}\text { Resting Pulse } \\
\text { (beat/min.) }\end{array}$ & $95 \pm 11.7$ & $85.88 \pm 9.8$ & $104.00 \pm 3.1$ & 24.7 & ${ }^{* *}<0.01$ \\
\hline
\end{tabular}

ФNormal figures for (3-5 years) according to PALS Guidelines, 2015 ( SBP:89-112(mm Hg) , DBP:46-72 (mm Hg) , Resting Pulse:80120 (beat/min.)

ФVo2 max. (ml/ $/ \mathrm{Kg} / \mathrm{min}): 51.3 \pm 8.9$ for boys \& $41.49 \pm 6.51 \mathrm{~mL} / \mathrm{kg} / \mathrm{min}$ in girls(Daniel et al.,2015)

Table (3) shows lower (IVC),(FVC),(FEV1),(PEF)\&(VO 2 max) among obese group in comparison to other groups. Also, they shows the highest (SBP),(DBP),(MAP) \& resting pulse, that means that obese group was the worst regarding cardio-respiratory fitness.

Table (3): Comparison between different categories of BMl regarding Cardio- respiratory measurements .

\begin{tabular}{|l|c|c|c|c|c|}
\hline Variables & $\begin{array}{c}\text { Normal } \\
\text { weight(121) }\end{array}$ & $\begin{array}{c}\text { Under } \\
\text { weight(21) }\end{array}$ & $\begin{array}{c}\text { Over- } \\
\text { weight(86) }\end{array}$ & $\begin{array}{c}\text { Obese } \\
\text { (172) }\end{array}$ & $\begin{array}{l}\text { P value } \\
\text { of f-test }\end{array}$ \\
\hline IVC & $1.9 \pm 0.3$ & $2.03 \pm 0.2$ & $1 \pm .07$ & $0.67 \pm 0.09$ & ${ }^{* *}<0.00$ \\
\hline FVC & $1.65 \pm 0.2$ & $1.7 \pm 0.08$ & $1 \pm 0.05$ & $0.8 \pm 0.09$ & ${ }^{* *}<0.00$ \\
\hline FEV 1 & $1.47 \pm 0.19$ & $1.5 \pm 0.11$ & $0.91 \pm 0.08$ & $0.74 \pm 0.11$ & ${ }^{* *}<0.00$ \\
\hline PEF & $1.69 \pm 0.22$ & $1.82 \pm 0.08$ & $1.2 \pm 0.08$ & $1 \pm 0.08$ & ${ }^{* *}<0.00$ \\
\hline $\mathrm{Vo}_{2}$ max. & $25.35 \pm 1.5$ & $26 \pm 0.83$ & $20 \pm 0.76$ & $17.11 \pm 0.89$ & ${ }^{* *}<0.00$ \\
\hline $\begin{array}{l}\text { SBP }(m m \\
\text { Hg) }\end{array}$ & $96.17 \pm 2.6$ & $95.04 \pm 1.1$ & $104 \pm 0.8$ & $106.9 \pm 0.9$ & ${ }^{* *}<0.00$ \\
\hline $\begin{array}{l}\text { DBP (mm } \\
\text { Hg) }\end{array}$ & $65.66 \pm 1.95$ & $64.95 \pm 1.4$ & $71.44 \pm 1.0$ & $74.9 \pm 0.96$ & ${ }^{* *}<0.00$ \\
\hline $\begin{array}{l}\text { MAP(mm } \\
\text { Hg) }\end{array}$ & $75.8 \pm 2.1$ & $74.97 \pm 1.2$ & $82.28 \pm 0.7$ & $85.56 \pm 0.95$ & ${ }^{* *}<0.00$ \\
\hline $\begin{array}{l}\text { Resting } \\
\begin{array}{l}\text { Pulse } \\
\text { (beat/min.) }\end{array}\end{array}$ & $80.47 \pm 5.6$ & $79.33 \pm 2.9$ & $99.67 \pm 1.8$ & $104.66 \pm 2.9$ & ${ }^{* *}<0.00$ \\
\hline
\end{tabular}

Table (4) shows strong inverse relationship between body fatness and respiratory function tests on one hand and strong direct relationship with SBP,DBP,MAP \& resting pulse on the other hand $(p<0.01)$. On the contrary there was positive correlation between body water content and (IVC),(FVC)\& $\left(\mathrm{VO}_{2} \max \right)$ and negative correlation with (SBP),(DBP),(MAP)\& resting pulse $(p<0.05)$. No significant association could be found between BMI and any of CRF measurements.

Table (4): Correlation between Body Composition measurements(BC) \& Cardio- Respiratory Fitness(CRF) of the total group 


\begin{tabular}{|c|c|c|c|c|c|c|c|c|}
\hline BC & Age & Weight & Height & Waist & Hip & $\begin{array}{l}\text { Body } \\
\text { fat }\end{array}$ & Fat \% & BMI \\
\hline$(\mathrm{IVC})_{(\mathrm{L})}$ & 0.024 & ${ }^{* \star} 0.56$ & $0.01-$ & ${ }^{\star *} 0.36$ & ${ }^{* *} 0.35$ & ${ }^{* \star} 0.26$ & ${ }^{\star \star} 0.41-$ & ${ }^{\star \star} 0.77$ \\
\hline$(\mathrm{FVC})_{(\mathrm{L})}$ & -0.007 & ${ }^{* *} 0.57$ & $0.03-$ & ${ }^{* *} 0.35$ & ${ }^{* *} 0.36$ & ${ }^{* *} 0.26$ & ${ }^{\star *} 0.42$ & ${ }^{* *} 0.76-$ \\
\hline $\mathrm{FEV}_{1(\mathrm{~L})}$ & 0.005 & ${ }^{* *} 0.55$ & 0.003 & ${ }^{* *} 0.34$ & ${ }^{* *} 0.35$ & ${ }^{\star *} 0.26$ & ${ }^{\star \star} 0.41-$ & ${ }^{\star \star} 0.76$ \\
\hline PEF & 0.033 & ${ }^{* *} 0.54$ & 0.007 & ${ }^{* *} 0.33$ & ${ }^{* *} 0.33$ & ${ }^{* *} 0.26$ & ${ }^{\star *} 0.40$ & ${ }^{\star *} 0.76-$ \\
\hline $\begin{array}{l}\mathrm{Vo}_{2} \max \\
(\mathrm{ml} / \mathrm{Kg} / \mathrm{min})\end{array}$ & 0.029 & ${ }^{* *} 0.59$ & $0.016-$ & ${ }^{* \star} 0.36$ & ${ }^{* \star} 0.34$ & **0.27 & ${ }^{\star *} 0.44$ & ${ }^{\star \star} 0.79$ \\
\hline $\begin{array}{l}\text { SBP }(\mathrm{mm} \\
\mathrm{Hg})\end{array}$ & $0.013-$ & ${ }^{* *} 0.58$ & 0.012 & ${ }^{* *} 0.34$ & ${ }^{* *} 0.35$ & ${ }^{\star *} 0.25$ & ${ }^{* \star} 0.41$ & ${ }^{* *} 0.78$ \\
\hline $\begin{array}{l}\mathrm{DBP}(\mathrm{mm} \\
\mathrm{Hg})\end{array}$ & $0.038-$ & ${ }^{* *} 0.57$ & $0.001-$ & ${ }^{* *} 0.34$ & ${ }^{* *} 0.32$ & ${ }^{\star *} 0.27$ & ${ }^{* *} 0.43$ & ${ }^{* *} 0.79$ \\
\hline $\mathrm{MAP}(\mathrm{mmHg})$ & 0.029 & **058 & 0.004 & ${ }^{* *} 0.34$ & ${ }^{* *} 0.34$ & ${ }^{\star *} 0.26$ & ${ }^{* *} 0.43$ & ${ }^{* \star} 0.79$ \\
\hline $\begin{array}{l}\text { Resting } \\
\text { Pulse } \\
\text { (beat/min.) }\end{array}$ & 0.004 & ${ }^{\star \star} 0.56$ & 0.005 & ${ }^{\star \star} 0.34$ & ${ }^{* \star} 0.37$ & ${ }^{\star \star} 0.25$ & ${ }^{* \star} 0.42$ & ${ }^{\star *} 0.77$ \\
\hline
\end{tabular}

Table (5): Correlation between Body Composition measurements(BC) \& Cardio- Respiratory Fitness(CRF) of the male \& female

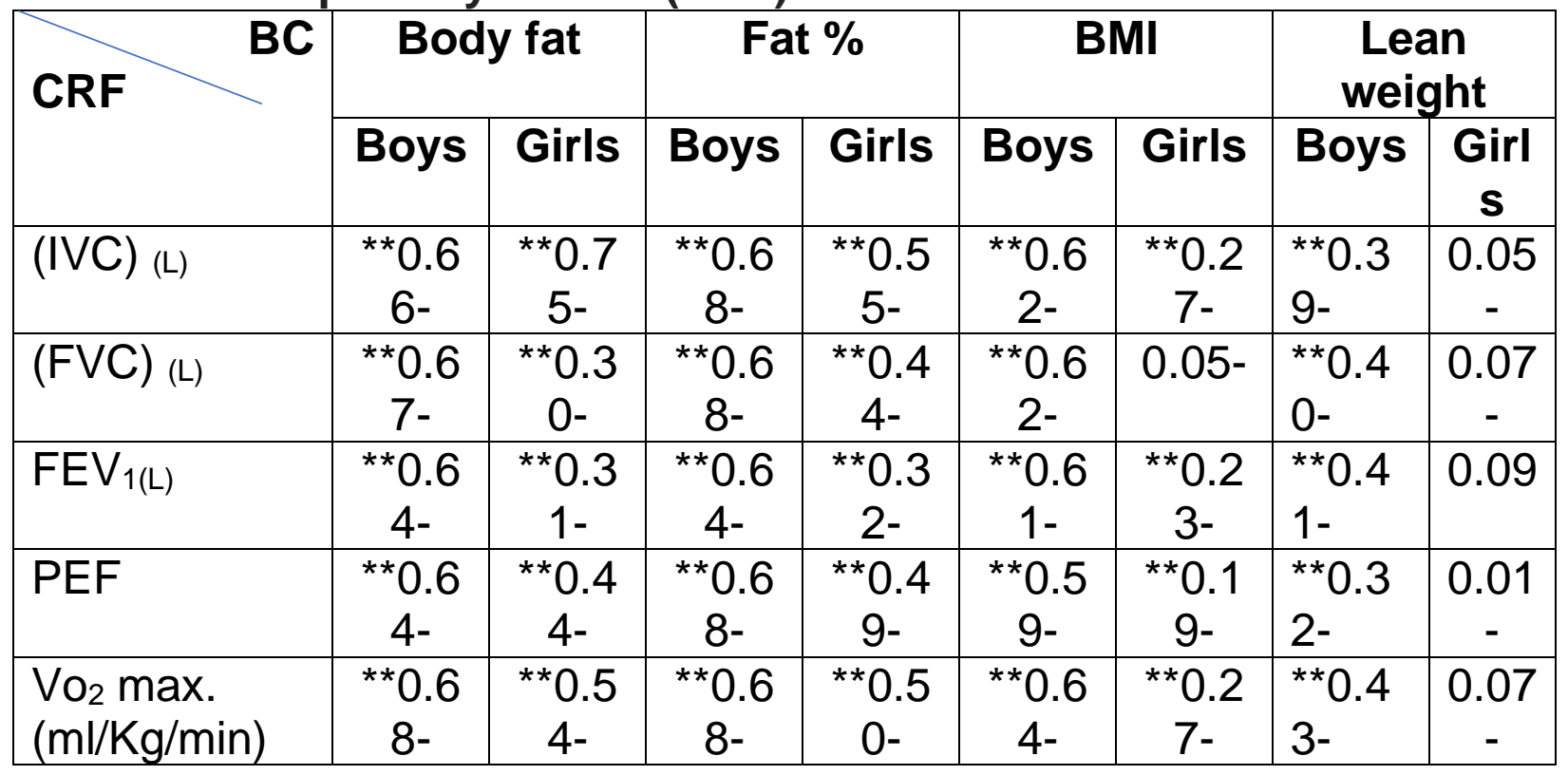




\begin{tabular}{|c|c|c|c|c|c|c|c|c|}
\hline $\begin{array}{l}\text { SBP }(\mathrm{mm} \\
\mathrm{Hg})\end{array}$ & $\begin{array}{l}{ }^{* *} 0.6 \\
6\end{array}$ & $\begin{array}{c}* * 0.8 \\
4\end{array}$ & $\begin{array}{c}{ }^{* *} 0.6 \\
8\end{array}$ & $\begin{array}{c}{ }^{* *} 0.5 \\
1\end{array}$ & $\begin{array}{c}{ }^{* *} 0.6 \\
2\end{array}$ & $\begin{array}{l}{ }^{* *} 0.3 \\
2\end{array}$ & $\begin{array}{l}* \star \\
0\end{array}$ & 0.05 \\
\hline $\mathrm{DBP}(\mathrm{mm} \mathrm{Hg})$ & $\begin{array}{c}* * 0.6 \\
2\end{array}$ & $\begin{array}{c}* * 0.7 \\
7\end{array}$ & $\begin{array}{c}{ }^{* *} 0.6 \\
4\end{array}$ & $\begin{array}{c}{ }^{* *} 0.5 \\
7\end{array}$ & $\begin{array}{c}{ }^{\star \star} 0.6 \\
1\end{array}$ & $\begin{array}{c}{ }^{* *} 0.3 \\
5\end{array}$ & ${ }_{7}^{* *} 0.3$ & 0.07 \\
\hline $\operatorname{MAP}(\mathrm{mmHg})$ & $\begin{array}{c}{ }^{* *} 0.6 \\
5\end{array}$ & $\begin{array}{c}{ }^{\star *} 0.8 \\
0\end{array}$ & $\begin{array}{l}{ }^{* \star} 0.6 \\
7\end{array}$ & $\begin{array}{c}{ }^{* *} 0.5 \\
6\end{array}$ & $\begin{array}{c}{ }^{\star \star} 0.6 \\
2\end{array}$ & $\begin{array}{l}{ }^{* *} 0.3 \\
5\end{array}$ & $\begin{array}{l}{ }^{* *} 0.3 \\
8\end{array}$ & 0.07 \\
\hline $\begin{array}{l}\text { Resting } \\
\text { pulse(beat/mi } \\
\text { n.) }\end{array}$ & $\begin{array}{l}{ }^{* *} 0.6 \\
7\end{array}$ & $\begin{array}{c}{ }^{* *} 0.2 \\
7\end{array}$ & $\begin{array}{c}{ }^{* \star} 0.6 \\
8\end{array}$ & $\begin{array}{c}{ }^{* \star} 0.2 \\
7\end{array}$ & $\begin{array}{l}{ }^{* \star} 0.6 \\
2\end{array}$ & $\begin{array}{l}{ }^{* \star} 0.2 \\
2\end{array}$ & $\begin{array}{l}{ }^{* \star} 0.3 \\
9\end{array}$ & 0.04 \\
\hline
\end{tabular}

Table(6): Multiple linear regression analysis predictors of $\mathrm{VO}_{2} \max \&$ MAP for the total sample.

\begin{tabular}{|c|c|c|c|c|c|c|}
\hline \multirow[b]{2}{*}{ Model } & \multicolumn{3}{|c|}{ (1) $\mathrm{VO}_{2} \max$} & \multicolumn{3}{|c|}{ (2) MAP } \\
\hline & Beta & $t$ & $\mathbf{P}$ & Beta & $t$ & $\mathbf{P}$ \\
\hline Constant & 55.3 & 19.87 & ${ }^{* *}<0.000$ & 41.3 & 12.38 & ${ }^{* *}<0.000$ \\
\hline Waist & 0.021 & 0.475 & $>0.05$ & -0.048 & 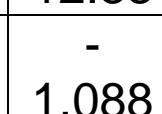 & $>0.05$ \\
\hline $\begin{array}{c}\text { Hip } \\
\text { circumference }\end{array}$ & -0.056 & -1.14 & $>0.05$ & 0.075 & 1.522 & $>0.05$ \\
\hline Body fatness & -0.044 & -0.609 & $>0.05$ & 0.066 & 0.902 & $>0.05$ \\
\hline Lean weight & -0.038 & $\mid-0.761$ & $>0.05$ & 0.015 & 0.300 & $>0.05$ \\
\hline BMI & -0.667 & -11.46 & ${ }^{* *}<0.000$ & 0.669 & 11.43 & ${ }^{* *}<0.000$ \\
\hline Fat $\%$ & -0.156 & -4.82 & ${ }^{* *}<0.000$ & 0.140 & 4.27 & ${ }^{* *}<0.000$ \\
\hline $\mathrm{R}=0.81$ & $=0.656$ & $\mathrm{SEE}=2$ & & & .000 & \\
\hline
\end{tabular}

Discussion:

Early childhood has emerged as a critical period for assessing the beginnings of obesity and instituting preventive measures; given the evidence linking excessive weight gain in the first years of life to obesity and chronic disease in later years ${ }^{26}$

The current study examined the BC of pre-school children and its association with CRF. The anthropometric measures of our sample showed that girls were significantly taller with more body fat and fat \%,while boys had more lean weight, dry lean, dry lean \% and water content. No significant differences could be found between both sexes regarding body weight \& BMI. Similar results were obtained by Tanaka et al., $2012^{27}$ and Vameghi et al., $2013^{28}$.

According to BMI classification, we found that (43\% obese, $21.5 \%$ over-weight , $5.3 \%$ under-weight $\& 30.2 \%$ normal weight) . Our findings did 
not come in agreement with another Egyptian study conducted in Alexandria on similar age group, their findings were ( $23 \%$ obese , $14 \%$ overweight, $6 \%$ underweight and $57 \%$ normal weight for age) ${ }^{29}$. A similar study conducted on 500 children, 2-5 years old, in kindergartens of Birjand, Iran; found that prevalence of obesity $(7.6 \%)$ \& overweight $(10.6 \%)^{30}$. Our findings were higher than previous studies due to lack of nutritional awareness \& physical activity in the community of the study. Gender specific prevalence of obesity in our study was (54.7\% for boys vs. $45.3 \%$ for girls) ,over-weight (36\% for boys vs. $64 \%$ for girls). That means boys were more obese, while girls were more over-weight. These findings agreed with the national data ${ }^{5}$. and with other several studies in Alexandria ${ }^{29}$,China ${ }^{31}$, Iran $^{30}$, Bahrain ${ }^{32}$ and Kuwait ${ }^{33}$. According to a systematic review by Musaiger AO, the prevalence of overweight among preschool children of the EMR countries ranged from $1.9 \%$ to $21.9 \%$. Predicting factors determining obesity in this region are frequent snacking, and massive marketing promotion of high fat foods ${ }^{34}$.

Our study found a significant differences between boys and girls regarding CRF measurements. Boys had better findings than girls but still all findings below normal for this age group, that is mean poor CRF of preschool children. Our findings came in agreement with Pedro et al., 2016 ${ }^{35}$. The aim of his study was to determine the physical fitness in children 3 - 6 years old, discriminating performance by sex, age and BMI. His main finding was that sex differences in physical fitness were evident at an early age. These differences could be explained because of the fact that preschool girls present a more sedentary lifestyle and lower levels of moderate to vigorous physical activity than boys ${ }^{27}$

Boys showed significantly higher (IVC), (FVC), (FEV1) and (PEF) than girls. Also, $\left(\mathrm{VO}_{2} \max \right)$ which was found to be the most widely used indicator of CRF, was significantly higher among boys but still below normal for the same age group.

The results obtained from simple correlation coefficients indicate a significant negative correlation between ( weight, waist circumference, hip circumference, BMI, body fat, fat \%) and PFTs (IVC, FVC,FEV 1 , PEF \& $\mathrm{VO}_{2} \max$ ). Correlation Coefficients ( $\mathrm{r}$ ) for (body fat, fat \%, BMI ) and ( $\mathrm{FVC}$, $\mathrm{FEV}_{1}$, PEF \& $\left.\mathrm{VO}_{2} \max \right)$ were higher for boys than for the girls except for (IVC). These results supports the findings of the study by de Andrade Goncalves et al., $2017^{36}$.No significant correlation could be detected between lean weight in girls \& PFTs.

On contrary, girls had significantly higher (SBP, DBP , MAP\& Resting Pulse) than boys. Similar study ${ }^{37}$ was done in Spain for estimation of prevalence of high blood pressure in children $<6$ years old, the mean SBP 
of total group was $(102 \pm 10.2$ ) and this was similar to our findings ( $102.4 \pm$

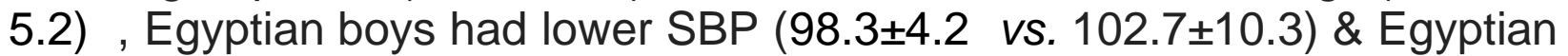
girls had higher SBP (106.5 \pm 1.4 vs. 101.6 \pm 9.9$)$.Regarding DBP, the mean DBP for the total Spanish group and that of boys \&girls was lower than Egyptian children ( $62.3 \pm 8.5$ vs. $70.8 \pm 4.4$ for total group, $61.68 \pm 8.26$ vs. $67.3 \pm 3.1$ for boys $\& 62.89 \pm 8.74$ vs. $74.4 \pm 1.5$ for girls). Similarly, MAP of Spanish children was lower than Egyptian children whether the total mean or means of boys \& girls ( $75.55 \pm 8.36$ vs. $81.4 \pm 4.6 / 75.33 \pm 8.21$ vs. $77.6 \pm 3.5$ / $75.78 \pm 8.50$ vs. $85.1 \pm 1.4$ ).

According to BMI classification; (SBP, DBP \& MAP) were significantly higher among Spanish obese children in comparison to other BMI categories and this is consistent with our findings. Moreover Egyptian obese showed higher (DBP \& MAP) than Spanish ones (74.9 \pm 0.96 vs. $67.43 \pm 0.47$ \& $85.56 \pm 0.95$ vs. \& 81.92 \pm 0.67$)$. When investigating the correlation between BC parameters \& (SBP, DBP, MAP \& Resting Pulse ), we found a strong positive relationship. Correlation Coefficients $(r)$ for boys were higher except for the relation between body fat and (SBP, DBP \& MAP). The relationship between adiposity with different body compositions in children has been shown in several studies. Eisenmann et al.,2005 38 reported that BMI, WC, sum of skinfolds and \%FM (measured via dual energy X-ray absorptiometry) were moderately and positively correlated with SBP, DBP and MAP, as found in other studies in which BMI were associated with SBP and DBP.

Our results, are in line with other studies, supporting that children with more adiposity are more likely to have higher risk of hypertension. Our findings also suggest that children in the higher categories of BMI and \%FM have higher levels of SBP, DBP, MAP in both boys and girls, as reported in other studies in the same age group and different ethnicities ${ }^{39-43}$

Multilinear regression analysis revealed that only BMI \& fat \% are the significant predictors of CRF . Our findings are supported with Maciejczyk et al., $2014^{18}$ and Ortega et al., $2008^{22}$,they stated that obese and overweight persons, whose high BM is caused by high body adiposity, display a considerably lower $\mathrm{VO}_{2}$ max relative to their $\mathrm{BM}$. Also, reduced $\mathrm{VO}_{2} \max$ as $\mathrm{BMI}$ increased. The negative impact of obesity on physical fitness has been documented in youth. There are inverse relationships between physical fitness and overweight ${ }^{37}$. However, Lopes et al., $2011^{44}$ found no correlation between BMI and physical fitness. Hence, the data on the relationship between physical fitness and BMI are inconsistent in preschool children. However, we indicated that BMI alone could not be considered as an indicator for physical fitness in preschool-age children. BMI alongside fat \% are important indicator for CRF. 


\section{Conclusions:}

1. Pre-school children under study suffer from increased physical composition ( weight, Fat Mass, waist / hip ratio \& BMI ) in comparison to reference measurements. Also we found a decrease in the value of FatFree Mass (Lean Weight).

2. $69.8 \%$ of pre-school children are of abnormal weight $(43 \%$ obese, $21.5 \%$ over-weight \& 5.3\% under-weight).

3. Poor CRF of pre-school children, whether respiratory-related measures such as (VC, FVC, FEV1, the PEF and VO2max).

4. Strong inverse correlation between body composition measurements like ( body fat, BMI \& Waist /hip ration) and respiratory fitness measurements like (VC, FVC, FEV1, the maximum effluent flow and maximum oxygen consumption).On the contrary, there is strong direct correlation between body composition and SBP \& DBP at rest \& resting pulse rate).

\section{References:}

1. World Health Organization reports. Preventing chronic diseases, a vital investment. Geneva, 2005.

2. World Health Organization, Obesity and overweight fact sheet. Accessed in August 2016. http://www.who.int/mediacentre/factsheets/fs311/en/

3. World Health Organization, Obesity and overweight fact sheet. Accessed in 16 February

2018. http://www.who.int/mediacentre/factsheets/fs311/en/

4. Musaiger AO. Overweight and Obesity in Eastern Mediterranean Region: Prevalence and Possible Causes. J Obesity 2011; 2011: 1- 18.

5. UNICEF Children in Egypt 2016: a statistical digest. The Central Agency for Public Mobilization and Statistics (CAPMAS), Egypt-United Nations Children's Fund (UNICEF), Egypt, January 2017.

6. Line T, Céline Boudreau L and Krystel CL. Promoting Physical Activity in Preschoolers: A Review of the Guidelines, Barriers, and Facilitators for Implementation of Policies and Practices.2012.

7. de Andrade Goncalves EC, Nunes HEG, \& Silva DAS. Which body fat anthropometric indicators are most strongly associated with maximum oxygen uptake in adolescents?. Asian Journal of Sports Medicine .2017, 8(3): e13812.

8. Wedderkopp N, Froberg K, Hansen HS, Andersen LB, Scand. J Med Sci Sports, 14 (2004) 150.

9. Hansen SE, Hasselstrom H, Gronfeldt V, Froberg K, Anbdersen LB. Prev Med, 40 (2005) 740.

10. Ribeiro JC, Guerra S, Oliveira J, Andersen LB, Duarte JA, Mota J. Am J Hum Biol, 16 (2004) 556. 
11. Webster-Gandy J, Warren J, Henry CJ. Int J Food Sci Nutr, 54 (2003) 467.

12. Poplawska H, Dmitruk A, Wilczewski A. Ann Hum Biol, 2006; 33(1): 78-88.

13. Orden AB and Oyhenat EE. Am J Hum Biol, 18 (2006) 590.

14. Lukaski HC, Johnson PE., Bolonchuk WW, \& Lykken Gl. Assessment of fat-free mass using bioelectrical impedance measurements of the human body. The American journal of clinical nutrition. 1985, 41(4), 810817.

15. Ranković G, Mutavdžić V, Toskić D, Preljević A, Kocić M, Nedin GR, \& Damjanović N. Aerobic capacity as an indicator in different kinds of sports. Bosnian Journal of Basic Medical Sciences. 2010, 10(1), 44-48.

16. Bassett DR \& Howley ET. Limiting factors for maximum oxygen uptake and determinants of endurance performance. Medicine and Science in Sports and Exercise. 2000, 32(1), 70-84.

17. Shete AN, Bute SS \& Deshmukh PR. A study of $\mathrm{VO}_{2} \mathrm{Max}$ and body fat percentage in female athletes. Journal of Clinical and Diagnostic Research: JCDR 2014, 8(12), BC01-3.

18. Maciejczyk M, Więcek M, Szymura J, Szyguła Z, Wiecha S \& Cempla $J$. The influence of increased body fat or lean body mass on aerobic performance. PloS One 2014, 9(4), e95797

19. Gligoroska JP, Manchevska S, Efremova L, Todorovska L, \& Nikolić S. Body composition and maximal oxygen consumption in adult soccer players in the Republic of Macedonia. Journal of Health Sciences.2015, 5(3), 85-92.

20. León-Ariza HH, Botero-Rosas DA \& Zea-Robles AC. Heart rate variability and body composition as $\mathrm{VO}_{2} \mathrm{max}$ determinants. Revista Brasileira de Medicina do Esporte .2017, 23(4), 317-321.

21. Tomassoni TL, Blanchard MS \& Goldfarb AH. Effects of a rebound exercise training program on aerobic capacity and body composition. The Physician and Sports medicine. 1985 13(11), 110-115.

22. Ortega FB, Ruiz JR, Castillo MJ \& Sjöström M. Physical fitness in childhood and adolescence: a powerful marker of health. International journal of obesity.2008, 32(1), 1-11.

23. McNaughton L, Hall P \& Cooley D. Validation of several methods of estimating maximal oxygen uptake in young men. Perceptual and Motor Skills . 1998, 87(2), 575-584.

24. Ramsbottom R, Brewer J \& Williams C. A progressive shuttle run test to estimate maximal oxygen uptake. British Journal of Sports Medicine. 1988, 22(4), 141-144. 
25. National institute for health research. Southampton Biomedical Research Centre. NIHR Southampton Biomedical Research Centre Procedure for using the BODYSTAT QUADSCAN 4000 bioelectrical impedance machine.

26. Institute of Medicine (IOM). Early Childhood Obesity Prevention Policies. Washington, DC: The National Academies Press, 2011.

27. Tanaka C, Hikihara Y, Ohkawara K \& Tanaka S. Locomotive and nonlocomotive activity as determined by triaxial accelerometry and physical fitness in Japanese preschool children. Pediatric Exercise Science. 2012,(24): 420-34

28. Vameghi R, Shams A \& Shamsipour Dehkordi P. The effect of age, sex and obesity on fundamental motor skills among 4 to 6 years-old children. Pakistan Journal of Medical Science. 2013(29), 586-9.

29. Dalia $T$ \& Amany S. Overweight and Obesity among Preschool Children aged 2-5 Years in Alexandria, Egypt. January. Canadian Journal of Clinical Nutrition. 2018, 6(1):34-54 .DOI: 10.14206/canad.j.clin.nutr.2018.01.04

30. Fatemeh T, Mohammad-Mehdi HT, Toba K, Afsaneh N \& Sharifzadeh G. Prevalence of overweight and obesity in preschool children (2-5 yearolds) in Birjand, Iran. BMC Research Notes 2012; 5: 25; 5:529. doi:10.1186/1756-0500-5-529.

31. Xiao Y, Qiao Y, Pan L, Liu J, Zhang T, Li N. Trends in the prevalence of overweight and obesity among Chinese preschool children from 2006 to 2014. PLoS ONE 2015; 10: e0134466.

32. Al Alawi S, Abdulatif $F$, Al Dhubaib D, Mohammed H, Al Ansari A. Prevalence of overweight and obesity across preschool children from four cities of the Kingdom of Bahrain. Int J Med Sci Public Health 2013; 2: 529532.

33. Amine EK. Nutritional Status Survey of Preschool Children in Kuwait. East Mediterr Health J 1996; 2: 386-395.

34. Musaiger AO. Overweight and Obesity in Eastern Mediterranean Region: Prevalence and Possible Causes. J Obesity 2011; 2011: 1- 18.

35. Latorre Román PÁ, Moreno del Castillo R, Lucena Zurita M , Salas Sánchez J , García-Pinillos and Mora López D. Physical fitness in preschool children: association with sex, age and weight status. Child:care, health and development. 2016 John Wiley \& Sons Ltd doi:10.1111/cch.12404.

36. de Andrade Goncalves EC, Nunes HEG, \& Silva DAS. Which body fat anthropometric indicators are most strongly associated with maximum 
oxygen uptake in adolescents?. Asian Journal of Sports Medicine.(2017), 8(3): e13812.

37. Noelia M , Ana D, Mairena S, Irene R, Lidia L, Montserrat S, Vicente M, Movi-Kids group. Prevalence of high blood pressure and association with obesity in Spanish schoolchildren aged 4-6 years old. PLOS ONE | DOI:10.1371/journal.pone.0170926 January 31, 2017.

38. Eisenmann JC, Wrede J, Heelan KA. Associations between adiposity, family history of CHD and blood pressure in 3-8 year-old children. J Hum Hypertens. 2005; 19(9): 675-81. doi: 10.1038/sj.jhh.1001882 PMID: 15905885

39. Falkner B, Gidding SS, Ramirez-Garnica G, Wiltrout SA, West D, Rappaport EB. The relationship of body mass index and blood pressure in primary care pediatric patients. J Pediatr. 2006; 148(2): 195- 200. doi: 10.1016/j.jpeds.2005.10.030 PMID: 1649242834.

40. Salvadori M, Sontrop JM, Garg AX, Truong J, Suri RS, Mahmud FH, et al. Elevated blood pressure in relation to overweight and obesity among children in a rural Canadian community. Pediatrics. 2008; 122(4): 821-7 35.

41. Flores-Huerta S, Klu"nder-Klünder M, Reyes de la Cruz L, Santos Jl. Increase in body mass index and waist circumference is associated with high blood pressure in children and adolescents in Mexico city. Arch Med Res. 2009; 40(3): 208-15. doi: 10.1016/j.arcmed.2009.02.009 PMID: 1942797336.

42. Almas A, Jafar TH. Adiposity and blood pressure in South Asian children and adolescents in Karachi. Am J Hypertens. 2011; 24(8): 87680. doi: 10.1038/ajh.2011.67 PMID: 2150905037.

43. LA de Hoog M, van Eijsden M, Stronks K, Gemke RJ, Vrijkotte TG. Association between body size and blood pressure in children from different ethnic origins. Cardiovasc Diabetol. 2012; 11:136. doi: 10. 1186/1475-2840-11-136 PMID: 23126496

44. Lopes VP, Rodrigues LP, Maia JAR. \& Malina RM. Motor coordination as predictor of physical activity in childhood. Scandinavian Journal of Medicine \& Science in Sports.2011, 21, 663-9. DOI:10.1111/j.16000838.2009.01027.x. 